\title{
On the Relationship Between Relay and Infrastructure Densities in Geometrically Bounded Relay-Assisted Wireless Networks
}

\author{
Dene A. Hedges and Justin P. Coon, Senior Member, IEEE
}

\begin{abstract}
In this paper, we study the connectivity between two fixed terminal nodes within a geometrically bounded network of relay nodes and fixed infrastructure nodes. We assume a communication path, between the terminal nodes, can be established by direct connection (1-hop), via a relay node (2-hop) or via the infrastructure network; the choice is based on link viability in a Rayleigh fading environment. We adopt a probabilistic approach to our analysis considering a homogeneous spatial distribution of relay nodes and an inhomogeneous spatial distribution of infrastructure nodes. Our analysis of the relationship between relay and infrastructure densities for a prescribed outage probability shows that reliance on infrastructure connectivity can be appreciably reduced by employing direct and 2-hop connectivity. We further show that, as the relay density increases, optimum connectivity is achieved by employing a non uniform spatial thinning of the infrastructure, which is dependent upon the geometric extent of the network. Our analysis provides an insight into multi-mode connectivity in bounded network domains and has application in many fields including cellular and vehicle-to-infrastructure (V2X), which are often modelled as having finite extent and where reducing the reliance on fixed infrastructure can effectively reduce operating expenditure (OPEX) costs.
\end{abstract}

Index Terms-2-hop connectivity, cooperative networks, stochastic geometry.

\section{INTRODUCTION}

$\mathbf{I}$ $\mathrm{N}$ the context of evolution towards 5 th generation wireless systems $(5 \mathrm{G})$, network densification [1] is considered a key enabler in satisfying the increasing capacity demands on wireless communication networks, whilst maximising spectrum utilisation [2]. The past few decades have seen the traditional macro-cellular networks being augmented with tiered supplemental infrastructures in the forms of micro, pico and femto-cells. The gains and limitations associated with the employment of these denser smaller cell networks are discussed in [3] and references therein. As we move down the tiered structure of the network, from the macro layer, we observe three general trends: the location of cells migrates from being planned to unplanned (spatially random), energy consumption of the cell decreases and cell densities (number of active nodes per unit area) increase [4] [5]. The $5 \mathrm{G}$ picture is further complicated by the integration of a

Copyright (c) 2015 IEEE. Personal use of this material is permitted. However, permission to use this material for any other purposes must be obtained from the IEEE by sending a request to pubs-permissions@ieee.org.

D.A. Hedges and J. P. Coon are with the Department of Engineering Science, University of Oxford, Oxford OX1 3PJ, U.K. (e-mail: dene.hedges@eng.ox.ac.uk; justin.coon@eng.ox.ac.uk).

This work was supported by EPSRC under Grant EP/N002350/1 (Spatially Embedded Networks). number of disparate technologies, such as Internet of Things (IoT), Wireless Sensor Networks (WSN) and Wireless Local Area Networks (WLAN), to provide seamless 'always-on' data transfer through the composite $5 \mathrm{G}$ network by choosing communication paths that optimise total network performance [6] [7].

Energy efficiency in future wireless networks is also a growing concern for network operators in response to global pressure to reduce carbon footprints and maintain profitability, with the term 'green cellular network' typically employed to convey an energy efficient network. [8] provides a cost-capacity performance analysis of femto-cell and macro-cellular networks, concluding that the optimum network structure depends on capacity demands. [9] provides a quantitative analysis of energy savings in green cellular networks and [10] provides a survey of research issues and challenges associated with managing energy consumption. A consensus is that the capital expenditure (CAPEX), costs associated with commissioning an infrastructure, and operating expenditure (OPEX), ongoing cost associated with running the infrastructure, are dominant in macro-cells but the costs associated with lower tier cells cannot be ignored due to their significantly higher spatial densities compared to macro-cell network. Recent research has concluded that the most efficient way to save energy in cellular networks is to switch base stations on and off dynamically according to the distribution of user equipment in real time. This is often termed 'Idle Mode' capability or 'Basestation Sleeping' and a number of strategies, and their effects on network performance, have been studied, including [11]-[14].

Another method to offload traffic from a network infrastructure is to allow users within range of each other to communicate directly or via a multi-hop route, through other user equipment. Spatial densification of user equipment (UE), e.g. mobile phones, is clearly beneficial in this context. Direct connection of proximity UEs, often termed device-to-device (D2D), has gained rapid traction over the past few years [15], [16]. Looking ahead, [17] predicts that few-hop networking is likely to play a key role in future wireless networks; optimised towards as few hops as possible in order to minimise end-to-end delivery latency and system complexity. Although significant research on this subject has been undertaken in geometrically unbounded networks, few papers have addressed the effects associated with finite geometries. [18], [19] and references therein provide useful insights into the effects of geometrically bounding wireless networks and [20] provides closed form expressions for the 2-hop connection probability 
in networks having circular and rectangular boundaries. We chose a working assumption that wireless networks are finite (geometrically bounded) because (a) it is realistic (wireless networks are not infinite), (b) connectivity performance analysis is mathematically tractable and (c) it enables us to consider the effects of inhomogeneity on a large scale, which can be useful in practice.

The connectivity between any two devices, wishing to communicate directly, is governed by their spatial separation, channel fading and a number of other system constants: wavelength, signal-to-noise ratio (SNR), etc. By allowing the two nodes to communicate via an intermediate node, i.e. forming a 2-hop path, it is possible to increase the communication range and/or connection probability. However, since the intermediate nodes are randomly distributed within the domain, we need to consider their spatial distribution and the distributions of other system variables in order to accurately analyse connectivity performance. Further, in the finite domain, the effects of the domain boundary need to borne in mind. To aid connectivity analysis, in these types of stochastic networks, we use tools taken from the world of stochastic geometry [21].

In this paper, we consider the use of direct (1-hop) and 2-hop connectivity, between two terminal nodes wishing to communicate, as a mechanism to reduce the reliance on a fixed infrastructure. This allows infrastructure nodes to be switched off according to the density of relay nodes within the network. Our approach considers outage probability between terminal nodes as our connectivity metric, which we average over all possible spatial relay/infrastructure node configurations within the network. For tractability, we consider the case of a circularly geometrically bounded wireless network subject to Rayleigh fading and assume negligible inter-node interference. The consideration of interference is clearly of concern in many applications; however we maintain generality by assuming a suitable medium access control protocol (MAC) [22] is employed to ensure interference is prevented, e.g. frequency/time division multiple access (FDMA/TDMA). Under these working assumptions, we are able to offer several contributions:

1) we provide mathematical expressions involving wellunderstood special functions for the connectivity probability of two terminal nodes in the network;

2) we exploit this mathematical framework to demonstrate the trade-off in relay and infrastructure node densities for a prescribed level of connectivity;

3) we demonstrate that it is possible to obtain a Pareto frontier in the different node densities by appropriately thinning the active infrastructure set in an inhomogeneous manner.

The remainder of the paper is organised as follows. Section II details our system model. Section III derives mathematical expressions involving well-understood special functions for outage probability between fixed terminal nodes, and Section IV provides insights into the relative contributions of relay and infrastructure networks for a prescribed outage probability when averaged over all spatial relay/infrastructure/terminal node configurations. Conclusions are detailed in section V.

\section{Network Definitions And System Model}

We consider the connectivity between two terminal nodes, at fixed locations $\mathbf{p}_{1}$ and $\mathbf{p}_{2}$, within a circular domain $\mathcal{V} \subset \mathbb{R}^{2}$. Also contained within $\mathcal{V}$, are independent identically distributed (i.i.d.) relay nodes and i.i.d. infrastructure nodes, spatially distributed according to independent Poisson point processes (PPP), $\Phi_{R}$ and $\Phi_{I}$ respectively. Note that the spatial distributions of relay and infrastructure networks are not necessarily identical. We employ PPP in our analysis for mathematical tractability and because we do not assume prior knowledge of the number of nodes in the networks. We characterise our relay and infrastructure networks by their average node densities $\rho_{\mathrm{R}}$ and $\rho_{\mathrm{I}}$ respectively; with zero node densities in $\mathbb{R}^{2} \backslash \mathcal{V}$. It is worth noting the distinction between a binomial point process and a PPP in a finite domain $\mathcal{V}$ is that different realisations of the PPP consist of a different number of points having a Poisson distribution [23, sect. 2.4.2]. We assume a homogeneous spatial distribution of relay nodes whereas the infrastructure nodes can follow an inhomogeneous spatial distribution where the density $\rho_{\mathrm{I}}$ is, in general, dependent upon the position within $\mathcal{V}$, as we define later. We denote the location of any arbitrary node- $i$ by the vector $\mathbf{p}_{i}$ with index $i \in\left\{1,2, \ldots,\left(\Phi_{\mathrm{R}}(\mathcal{V})+\Phi_{\mathrm{I}}(\mathcal{V})+2\right)\right\}$, where $\Phi_{y}(\mathcal{V}) \sim \operatorname{Poisson}\left(\rho_{y}|\mathcal{V}|\right), y \in\{\mathrm{R}, \mathrm{I}\}$. Hereafter, we use $\mathbf{p}_{i}$ to represent both node- $i$ and its location interchangeably.

The direct communication path between any two nodes, at locations $\mathbf{p}_{i}$ and $\mathbf{p}_{j}$, is considered undirected with reciprocal channel gain and connected with probability

$$
H_{i j}=\mathbb{P}\left(\mathrm{SNR}_{i j} \cdot\left|h_{i j}\right|^{2} \geqslant \tau\right),
$$

where $\mathrm{SNR}_{i j}$ denotes the long term average signal-to-noise ratio, $h_{i j}$ is the small scale fading coefficient for single input single output (SISO) systems, with $\mathbb{E}\left|h_{i j}\right|^{2}=1$, and $\tau$ is the required minimum instantaneous SNR for signal detection. Assuming identical lossless antennas with non directive gain, $G$, in the $\mathbb{R}^{2}$-plane, we have from the well known modified Friis transmission formula that

$$
\mathrm{SNR}_{i j}=\frac{P G^{2}}{\sigma^{2}}\left(\frac{\lambda}{4 \pi}\right)^{2}\left\|\mathbf{p}_{i}-\mathbf{p}_{j}\right\|^{-\eta},
$$

where $\eta$ is the path loss exponent, $P$ is the transmit power, $\sigma^{2}$ represents the thermal noise variance at the receiving node, $\lambda$ is the wavelength of the RF carrier employed and $\|\cdot\|$ is the $l^{2}$ norm. For convenience, we consider a fixed connectivity scale $r_{0}$ and, hereafter, normalise all distance variables to this scale. Further, we define $r_{0}$ as the hard connection range [24], which is the Euclidean distance beyond which there is insufficient signal level to allow signal detection. $r_{0}$ is determined solely by large scale path loss and from (2)

$$
r_{0}^{\eta}=\frac{P G^{2}}{\tau \sigma^{2}}\left(\frac{\lambda}{4 \pi}\right)^{2} .
$$

Letting $r_{i j}$ denote the Euclidean distance between $\mathbf{p}_{i}$ and $\mathbf{p}_{j}$, normalised to $r_{0}$, we obtain

$$
H_{i j}=\mathbb{P}\left(\left|h_{i j}\right|^{2} \geqslant r_{i j}^{\eta}\right)
$$

For mathematical tractability we assume the small scale fading coefficient follows a Rayleigh distribution, $h_{i j} \sim \mathcal{C N}(0,1)$. It 


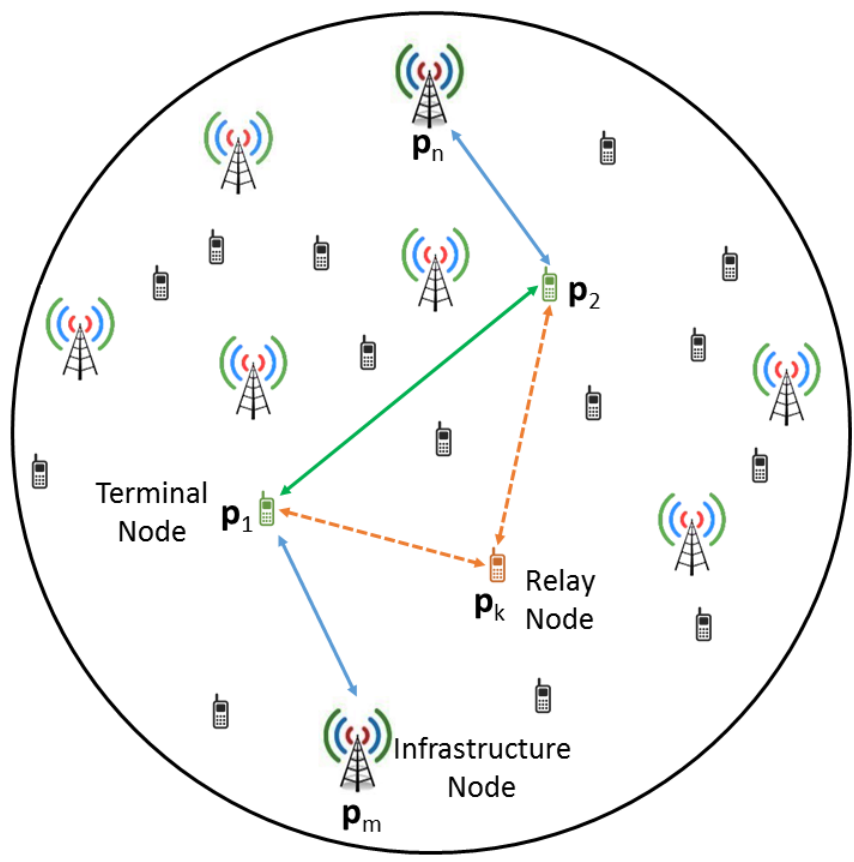

Fig. 1. Direct and 2-hop connectivity between two terminal nodes, $\mathbf{p}_{1}$ and $\mathbf{p}_{2}$, in a finite convex domain $\mathcal{V}$ containing uniformly distributed intermediate relay nodes $\mathbf{p}_{k} \in \Phi_{\mathrm{R}}$ and non-uniformly distributed infrastructure nodes $\mathbf{p}_{m}, \mathbf{p}_{n} \in \Phi_{\mathrm{I}}$.

then follows that $\left|h_{i j}\right|^{2}$ has a standard exponential distribution, $\left|H_{i j}\right|^{2} \sim \exp (1)$, which yields a connection probability defined by

$$
H_{i j}=e^{-r_{i j}^{\eta}}
$$

We consider three independent mechanisms whereby two terminal nodes $\mathbf{p}_{1}$ and $\mathbf{p}_{2}$ can connect: directly, via an intermediate node $\mathbf{p}_{k} \in \Phi_{\mathrm{R}}$ forming a 2-hop path, or independent connection to two infrastructure nodes $\mathbf{p}_{m}, \mathbf{p}_{n} \in \Phi_{\mathrm{I}}$, which is depicted in Fig. 1. Although our analysis is limited to the 2-hop relay case, K-hop connectivity could be considered [25]. The connectivity between any two infrastructure nodes is assumed to be guaranteed i.e. there exists some form of low latency fixed infrastructure with infinite capacity. For mathematical tractability, we assume that relaying nodes employ regenerative (decode-and-forward) relaying which eliminates interdependency on the connectivity of the 2-hops and allows the probability of 2-hop connectivity, between $\mathbf{p}_{1}$ and $\mathbf{p}_{2}$ via $\mathbf{p}_{k}$, to be simply expressed as the product $H_{1 k} H_{2 k}$. This also assumes that the small scale fading component of the 1-k and 2-k channels are statistically independent ${ }^{1}$.

\footnotetext{
${ }^{1}$ Although this is not necessarily the case when $\mathbf{p}_{1}$ and $\mathbf{p}_{2}$ are located within the coherence distance $r_{c}$ of each other since their connectivity behaviour to an arbitrary common relay node will be more highly correlated and therefore independence cannot be assumed. Taking the example of a circular domain $\mathcal{V}$ of radius $R$ and assume $r_{c} \ll R$, then the measure of space in $\mathcal{V}$ corresponding to the case $\left\|\mathbf{p}_{1}-\mathbf{p}_{2}\right\|<r_{c}$ is small compared to the whole space of node configurations. Therefore, if we average the exponential connectivity functions, between terminal nodes, over all possible node locations, then the portion of the average corresponding to $\left\|\mathbf{p}_{1}-\mathbf{p}_{2}\right\|<r_{c}$, will be negligible. This assumption has been shown on several occasions to yield accurate results [18] [26].
}

\section{Connectivity AnALYsis}

We choose outage probability as our connectivity metric between terminal nodes $\mathbf{p}_{1}$ and $\mathbf{p}_{2}$, for any spatial relay/infrastructure node configuration, denoted by $\mathcal{O}_{12}$. We assume the three aforementioned connection mechanisms are independent and evaluate $\mathcal{O}_{12}$ as the product of outage probabilities of all possible connection mechanisms ${ }^{2}$ such that

$$
\begin{array}{r}
\mathcal{O}_{12}=\left(1-H_{12}\right) \prod_{\mathbf{p}_{k} \in \Phi_{\mathrm{R}}}\left(1-H_{1 k} H_{2 k}\right) \\
\times\left[1-\left[1-\prod_{\mathbf{p}_{m} \in \Phi_{\mathrm{I}}}\left(1-H_{1 m}\right)\right]\left[1-\prod_{\mathbf{p}_{n} \in \Phi_{\mathrm{I}}}\left(1-H_{2 n}\right)\right]\right] .
\end{array}
$$

We now proceed to average the outage probability over all spatial relay/infrastructure node configurations within a finite circularly bounded domain $\mathcal{V}$ with radius $R$. A circularly bounded network is considered to be a reasonable approximation to a network constrained to a typical residential area or a clustering of communication nodes within a larger heterogeneous network for example. Alternative geometric boundaries, e.g. rectangular [20], can be treated in a similar manner to that outlined here. We firstly consider the simpler case of a homogeneous infrastructure network, also considering the special case $R \rightarrow \infty$, followed by analysis of an inhomogeneous infrastructure network. Closed form expressions are developed wherever possible to provide analytical insights.

\section{A. Homogeneous Infrastructure}

We define $\overline{\mathcal{O}}_{12}:=\mathbb{E}_{\Phi_{\mathrm{R}}, \Phi_{\mathrm{I}}}\left[\mathcal{O}_{12} \mid \mathbf{p}_{1}, \mathbf{p}_{2}\right]$ as the outage probability (6) averaged over all possible locations of relay and infrastructure nodes in $\mathcal{V}$, conditioned on the locations of the terminal nodes $\mathbf{p}_{1}$ and $\mathbf{p}_{2}$. Since relay and infrastructure node locations are independent and identically distributed (i.i.d.), this can be expressed as

$$
\begin{aligned}
\overline{\mathcal{O}}_{12} & =\left(1-H_{12}\right) \mathbb{E}_{\Phi_{\mathrm{R}}}\left[\prod_{\mathbf{p}_{k} \in \Phi_{\mathrm{R}}}\left(1-H_{1 k} H_{2 k}\right)\right] \\
& \times\left\{\mathbb{E}_{\Phi_{\mathrm{I}}}\left[\prod_{\mathbf{p}_{n} \in \Phi_{\mathrm{I}}}\left(1-H_{1 n}\right)\right]\right. \\
& +\left[\mathbb{E}_{\Phi_{\mathrm{I}}}\left[\prod_{\mathbf{p}_{n} \in \Phi_{\mathrm{I}}}\left(1-H_{2 n}\right)\right]\right. \\
& \left.-\mathbb{E}_{\Phi_{\mathrm{I}}}\left[\prod_{\mathbf{p}_{n} \in \Phi_{\mathrm{I}}}\left(1-H_{1 n}\right)\left(1-H_{2 n}\right)\right]\right\} .
\end{aligned}
$$

Employing the probability generating functional for $\Phi_{R}$ and $\Phi_{\mathrm{I}}$ to solve the expectations, gives

$$
\begin{array}{r}
\overline{\mathcal{O}}_{12}=\left(1-H_{12}\right) \exp \left[-\rho_{\mathrm{R}} V \bar{H}_{12}^{\left(2_{\mathrm{R}}\right)}\right] \\
\times\left\{\exp \left[-\rho_{\mathrm{I}} V \bar{H}_{1 \mathrm{I}}\right]+\exp \left[-\rho_{\mathrm{I}} V \bar{H}_{2 \mathrm{I}}\right]\right. \\
\left.-\exp \left[-\rho_{\mathrm{I}} V\left(\bar{H}_{1 \mathrm{I}}+\bar{H}_{2 \mathrm{I}}-\bar{H}_{12}^{\left(2_{\mathrm{I}}\right)}\right)\right]\right\},
\end{array}
$$

\footnotetext{
${ }^{2}$ We interpret connectivity, between terminal nodes, as a route exists in theory. The mathematical analysis is identical to the case of selection combining [27], although selection combining is impractical at a large scale.
} 
where $V=|\mathcal{V}|=\pi R^{2}$ and $\bar{H}_{12}^{\left(2_{y}\right)}, y \in\{\mathrm{R}, \mathrm{I}\}$, denotes the 2-hop connection probability, between $\mathbf{p}_{1}$ and $\mathbf{p}_{2}$ via a relay/infrastructure node, averaged over all possible locations of a relay/infrastructure node in $\mathcal{V} . \bar{H}_{i \mathrm{I}}, i \in\{1,2\}$, is the probability of a terminal node connecting directly to an infrastructure node, averaged over all locations of an infrastructure node in $\mathcal{V}$. Since we consider homogeneous relay and infrastructure networks,

$$
\bar{H}_{12}^{\left(2_{y}\right)}=\frac{1}{V} \int_{\mathcal{V}} H_{1 k} H_{2 k} \mathrm{~d} \mathbf{p}_{k},
$$

Note that the r.h.s. of (9) is independent of $y$ and the probabilities of terminal nodes connecting via a relay or infrastructure nodes are identical for the homogeneous spatial distribution of the infrastructure. The final term in the curly braces in (8) (similarly, (7)) is the probability of the union of two events, namely that each of the two terminal nodes does not connect to an infrastructure node. Hence, we can apply the union bound to simplify this expression. Intuitively, as the domain size increases, for a given $\rho_{I}$, the probability of the terminal nodes connecting to independent infrastructure nodes will be significantly higher than the probability of terminal nodes connecting to the same infrastructure node. Thus, we expect the union bound to provide a tight upper bound in outage probability. We denote this upper bound by $\overline{\mathcal{O}}_{12}^{\mathrm{UB}}$, where

$$
\begin{array}{r}
\overline{\mathcal{O}}_{12}^{\mathrm{UB}}:=\left(1-H_{12}\right) \exp \left[-\rho_{\mathrm{R}} V \bar{H}_{12}^{\left(2_{\mathrm{R}}\right)}\right] \\
\times\left\{\exp \left[-\rho_{\mathrm{I}} V \bar{H}_{1 \mathrm{I}}\right]+\exp \left[-\rho_{\mathrm{I}} V \bar{H}_{2 \mathrm{I}}\right]\right\} .
\end{array}
$$

Hereafter we chose the special case of free space propagation, $\eta=2$, to provide mathematical tractability in our analysis. Alternative path loss exponents could be employed but would not necessarily yield behavioural insights due to the limitations of a purely numeric analysis. (9) is evaluated in [20], for this special case, yielding

$$
\bar{H}_{12}^{\left(2_{y}\right)}=\frac{\pi}{2 V} e^{-\frac{r_{12}^{2}}{2}}\left[1-\mathrm{Q}_{1}\left(2\left\|\overline{\mathbf{p}}_{12}\right\|, 2 R\right)\right],
$$

where $\mathrm{Q}_{1}(a, b)=\int_{b}^{\infty} x e^{-\frac{\left(x^{2}+a^{2}\right)}{2}} \mathrm{I}_{o}(a x) \mathrm{d} x$ is the first order Marcum Q-function, $\mathrm{I}_{0}(a)=\frac{1}{\pi} \int_{0}^{\pi} e^{a \cos \theta} \mathrm{d} \theta$ is the zeroth order modified Bessel function of the first kind and $\overline{\mathbf{p}}_{12}=$ $\left(\mathbf{p}_{1}+\mathbf{p}_{2}\right) / 2$ is the mid-point between $\mathbf{p}_{1}$ and $\mathbf{p}_{2}$. Further, since we initially consider a uniform spatial distribution of infrastructure nodes,

$$
\bar{H}_{i I}=\frac{1}{V} \int_{\mathcal{V}} H_{i m} \mathrm{~d} \mathbf{p}_{m} .
$$

Using the approach detailed in [20], this evaluates to

$$
\bar{H}_{i I}=\frac{\pi}{V}\left[1-\mathrm{Q}_{1}\left(\sqrt{2} r_{i}, \sqrt{2} R\right)\right],
$$

where $r_{i}=\left\|\mathbf{p}_{i}\right\|$ is the radial offset of node $\mathbf{p}_{i}$.

Fig. 2 illustrates (8), and the upper bound (10), for a range of relay and infrastructure densities with fixed terminal nodes, located at polar coordinates $2 \angle 0$ and $2 \angle \pi / 2$, within a circular domain of radius 2.5 . The figure illustrates that

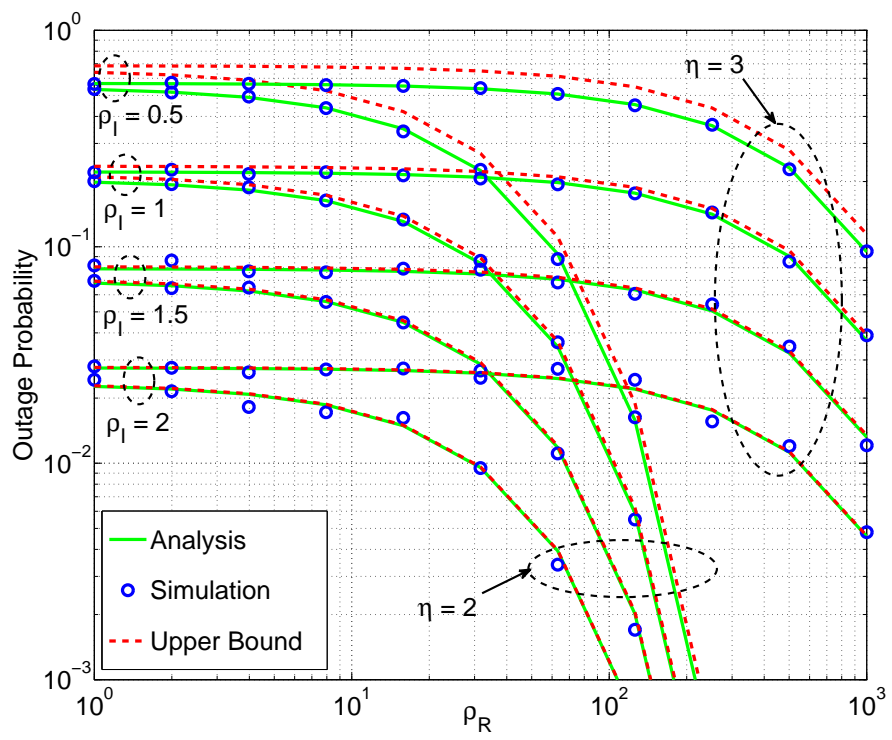

Fig. 2. Outage probability as a function of $\rho_{\mathrm{R}}$, for $\rho_{\mathrm{I}} \in\{0.5,1,1.5,2\}$ and $\eta \in\{2,3\}$, averaged over locations of relay and infrastructure nodes in a circular domain $\mathcal{V}$ of radius 2.5 , as given by (8). Terminal node located at polar coordinates $2 \angle 0$ and $2 \angle \pi / 2$. Blue markers show results from Monte Carlo simulations. Dashed red lines indicate upper bound as given by (10).

our expectation in upper bound tightness is indeed correct. Although we focus our analysis on free space propagation, we demonstrate the tightness of the bound for increased path loss exponent by including the case $\eta=3$, evaluating (9) and (12) numerically. Results from Monte Carlo simulations are included to validate the analysis. The figure clearly shows an exponential improvement in outage probability with increasing infrastructure density. Since $1-Q_{1}(x, \cdot)$ is strictly decreasing with respect to $x$, then (11) and (13) exhibit a reduction in connection probability as the terminal nodes move closer to the boundary of $\mathcal{V}$; following observations made in [19], [28] where this phenomena is attributed to loss in connectivity mass towards the boundary. We also observe a tight upper bound as the average number of infrastructure nodes increases. If we analyse the asymptotic behaviour of (11) and (13) with increasing $r_{12}$ and their relative contributions in (8), we observe that (13) becomes the dominant term due to the exponential term, yielding a tight upper union bound for larger terminal node separation or when we average connectivity over all possible terminal node positions with increasing domain size. Further, we can approximate performance with terminal node located towards the domain centre by employing the special cases for the first order Marcum Q-function

$$
\mathrm{Q}_{1}(0, x)=e^{-x^{2} / 2},
$$

and similarly at the boundary

$$
\mathrm{Q}_{1}(x, x)=\frac{1}{2}\left[1+e^{-x^{2}} \mathrm{I}_{0}\left(x^{2}\right)\right]
$$

which by taking the asymptotic expansion of the modified Bessel function for large argument $x$ becomes

$$
\mathrm{Q}_{1}(x, x) \sim \frac{1}{2}\left[1+\frac{1}{\sqrt{2 \pi}} \frac{1}{x}\right] .
$$




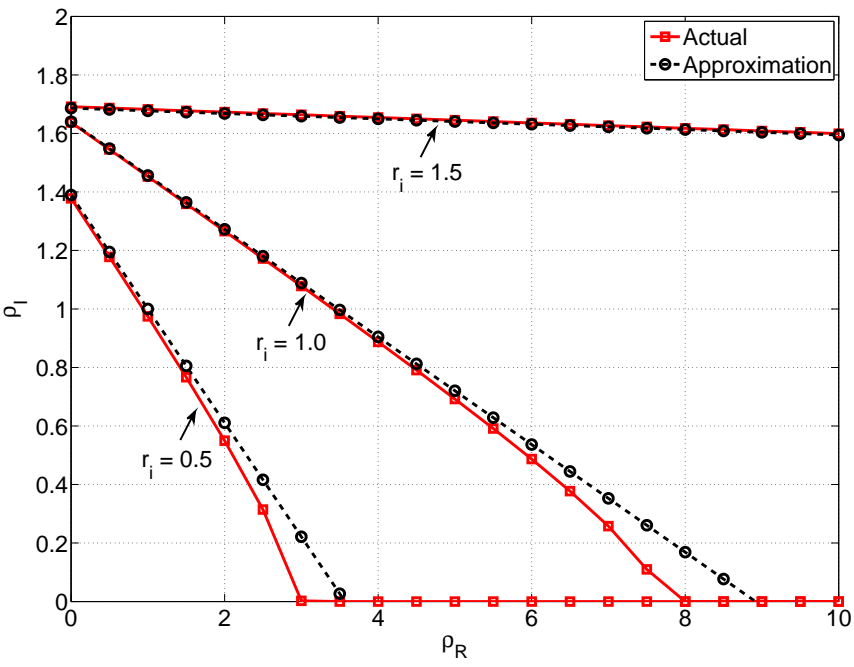

Fig. 3. Illustration of the accuracy of first order approximation (19). The red solid traces show $\rho_{I}$ as a function of $\rho_{R}$ as defined by (8) (determined through successive approximation) and the black dashed traces show the first order approximation as given by (19). We fix $R=4$ and $\kappa=0.01$ and consider terminal nodes located within the central bulk region at radial and angular offsets $r_{i} \in\{0.5,1.0,1.5\}$ and $\pi / 2$ respectively.

The effectiveness of these special cases for approximating performance at the centre and boundary of circular domains is demonstrated in [20]. From $\mathrm{Q}_{1}(x, \infty)=0$ [29] we can show that, in the limit $R \rightarrow \infty\left(\mathcal{V} \rightarrow \mathbb{R}^{2}\right)$, (8) reduces to

$$
\begin{array}{r}
\overline{\mathcal{O}}_{12} \underset{R \rightarrow \infty}{=}\left(1-e^{-r_{12}^{2}}\right) \exp \left(-\frac{\pi \rho_{\mathrm{R}}}{2} e^{-\frac{r_{12}^{2}}{2}}\right) \\
\times \exp \left(-\pi \rho_{\mathrm{I}}\right)\left[2-\exp \left[-\frac{\pi \rho_{\mathrm{I}}}{2}\left(2-e^{-\frac{r_{12}^{2}}{2}}\right)\right]\right]
\end{array}
$$

and similarly

$$
\overline{\mathcal{O}}_{12}^{\mathrm{UB}} \underset{R \rightarrow \infty}{=} 2\left(1-e^{-r_{12}^{2}}\right) \exp \left(-\frac{\pi \rho_{\mathrm{R}}}{2} e^{-\frac{r_{12}^{2}}{2}}\right) \exp \left(-\pi \rho_{\mathrm{I}}\right),
$$

which are solely functions of the Euclidean distance between the terminal nodes and the relay and infrastructure node densities. It is worth noting that the simplicity of (18) provides a convenient approximation for outage behaviour in the central bulk region $\left(r_{i}<0.5 R, i \in\{1,2\}\right)$ under the conditions $R>3$, yielding $0<\mathrm{Q}_{1}\left(\sqrt{2} r_{i}, \sqrt{2} R\right) \ll 0.1$ and $\rho_{I}>1$, yielding a tighter upper performance bound. Further, for a fixed $r_{12}$ and a prescribed outage probability $\overline{\mathcal{O}}_{12}^{\mathrm{UB}}=\kappa$, we can approximate the infrastructure density required for a given relay infrastructure by simply rearranging (18) such that

$$
\rho_{\mathrm{I}} \approx-\frac{1}{\pi} \ln \left[\frac{0.5 \kappa}{1-e^{-r_{12}^{2}}}\right]-0.5 e^{-\frac{r_{12}^{2}}{2}} \rho_{\mathrm{R}} .
$$

This accuracy of this approximation is illustrated in Fig. 3. If we wish to average connectivity performance over terminal node positions within the central bulk region we can employ the probability distribution of $r_{12}$ within a circle [30]. Further analysis in the bulk region of homogeneous networks has not been included for brevity; our main motivation is to understand the relationship between boundary conditions and spatial distribution of the infrastructure network.

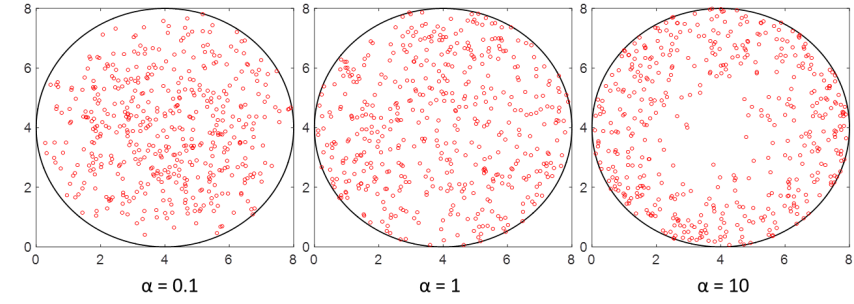

Fig. 4. Realisations of the spatial distribution of infrastructure nodes, with density $\rho_{\mathrm{I}}=10$ within a circle of radius $R=4$, for $\alpha \in\{0.1,1,10\}$ and $\beta=2$.

\section{B. Inhomogeneous Infrastructure}

Conjecturing that, for a non zero density of relays, an inhomogeneous distribution of infrastructure nodes will mitigate loss in relay assisted 2-hop connectivity between terminal nodes located closer to the boundary, we now consider an inhomogeneous independent thinning [23, section 2.7.3] of our infrastructure network as the density of the relay infrastructure increases. In the thinning process, we remove infrastructure nodes with probability $1-g\left(\mathbf{p}_{n}\right)$, independently of all other infrastructure nodes. This process generates an inhomogeneous PPP with intensity function $\rho_{\mathrm{I}} g\left(\mathbf{p}_{n}\right)$, where $\rho_{\mathrm{I}}$ is the mean infrastructure density over $\mathcal{V}$. We propose that $g\left(\mathbf{p}_{n}\right)$ has a uniform axial distribution but varies with radial offset $\left\|\mathbf{p}_{n}\right\|$ from the centre of $\mathcal{V}$ according to

$$
g\left(\mathbf{p}_{n}\right)=\frac{1}{k}\left[1+(\alpha-1)\left(\frac{\left\|\mathbf{p}_{n}\right\|}{R}\right)^{\beta}\right],
$$

where the normalising constant $k$ is given by

$$
\begin{aligned}
k & =\int_{\mathcal{V}}\left[1+(\alpha-1)\left(\frac{\left\|\mathbf{p}_{n}\right\|}{R}\right)^{\beta}\right] \mathrm{d} \mathbf{p}_{n} \\
& =\pi R^{2}\left[1+\frac{2(\alpha-1)}{\beta+2}\right] .
\end{aligned}
$$

The radial distribution of (20) is defined by parameters $\alpha$ and $\beta$. The exponent $\beta \in \mathbb{R}^{+}$defines the characteristic of the distribution, i.e. $\beta=1$ for a linear radial distribution and $\beta=2$ for a quadratic radial distribution. The parameter $\alpha \in \mathbb{R}^{+}$is a weighting factor given by the ratio of the intensity measures of infrastructure nodes at the domain's boundary and centre, i.e. $\alpha<1$ provides a spatial bias towards the centre of the domain, $\alpha>1$ spatially biases the infrastructure nodes towards the domain boundary and $\alpha=1$ yields a homogeneous spatial distribution. We illustrate the effect of this spatial bias in Fig. 4. Alternative radial distributions could have been considered, e.g. beta distribution, but do not necessarily yield the mathematical tractability offered by (20) or the spatial bias simply defined by a single parameter. We now evaluate (7), again employing the probability generating functional to solve the expectations. For the thinned infrastructure, we evaluate $\bar{H}_{i \mathrm{I}}$ and $\bar{H}_{12}^{\left(2_{\mathrm{I}}\right)}$ for the inhomogeneous spatial distribution.

$$
\bar{H}_{i \mathrm{I}}=\int_{\mathcal{V}} g\left(\mathbf{p}_{n}\right) H_{i n} \mathrm{~d} \mathbf{p}_{n},
$$


which, by employing polar coordinate geometry and firstly integrating over the axial component [20], results in

$\bar{H}_{i \mathrm{I}}=\frac{2 \pi}{k} e^{-r_{i}^{2}} \int_{0}^{R} r_{n} e^{-r_{n}^{2}}\left[1+(\alpha-1)\left(\frac{r_{n}}{R}\right)^{\beta}\right] \mathrm{I}_{0}\left(2 r_{i} r_{n}\right) \mathrm{d} r_{n}$,

where $r_{i}=\left\|\mathbf{p}_{i}\right\|$ is the radial offset of the terminal node and $r_{n}=\left\|\mathbf{p}_{n}\right\|$. Since (22) only produces a closed form expression at even integers for $\beta$ [31], we chose $\beta=2$. Since we are interested in observing the effect of this spatial bias this is considered a reasonable assumption. Splitting (22) and applying [31, eqs. (10) \& (20)] results in

$$
\begin{aligned}
\bar{H}_{i \mathrm{I}} & =\frac{\pi}{k}\left\{\left(\frac{(\alpha-1)\left(r_{i}^{2}+1\right)}{R^{2}}+1\right)\left[1-\mathrm{Q}_{1}\left(\sqrt{2} r_{i}, \sqrt{2} R\right)\right]\right. \\
& \left.-(\alpha-1) e^{-\left(r_{i}^{2}+R^{2}\right)}\left[\frac{r_{i}}{R} \mathrm{I}_{1}\left(2 r_{i} R\right)+\mathrm{I}_{0}\left(2 r_{i} R\right)\right]\right\} .
\end{aligned}
$$

Similarly

$$
\bar{H}_{12}^{\left(2_{1}\right)}=\int_{\mathcal{V}} g\left(\mathbf{p}_{n}\right) H_{1 n} H_{2 n} \mathrm{~d} \mathbf{p}_{n},
$$

which, employing a similar approach as before (see Appendix A), can be shown to yield

$$
\begin{aligned}
\bar{H}_{12}^{\left(2_{1}\right)} & =\frac{\pi}{2 k} e^{-\frac{1}{2} r_{12}^{2}} \\
& \times\left\{\left(\frac{(\alpha-1)\left(2\left\|\overline{\mathbf{p}}_{12}\right\|^{2}+1\right)}{2 R^{2}}+1\right)\right. \\
& \times\left[1-\mathrm{Q}_{1}\left(2\left\|\overline{\mathbf{p}}_{12}\right\|, 2 R\right)\right] \\
& -(\alpha-1) e^{-2\left(\left\|\overline{\mathbf{p}}_{12}\right\|^{2}+R^{2}\right)} \\
& \left.\times\left[\frac{\left\|\overline{\mathbf{p}}_{12}\right\|}{R} \mathrm{I}_{1}\left(4\left\|\overline{\mathbf{p}}_{12}\right\| R\right)+\mathrm{I}_{0}\left(4\left\|\overline{\mathbf{p}}_{12}\right\| R\right)\right]\right\} .
\end{aligned}
$$

It is trivial to show that a substitution of $\alpha=1$ into (23) and (24) reduces to (13) and (11) respectively, the homogeneous case.

We evaluate (8), following substitutions of (23) and (24), to yield outage probability averaged over all spatial node configurations of our homogeneous relay and inhomogeneous infrastructure networks, between fixed terminal nodes. This is illustrated in Fig. 5 for the case where the domain radius is $R=2.5$, terminal nodes are located at polar coordinates $2 \angle 0$ and $2 \angle \pi / 2$, infrastructure density is fixed at $\rho_{\mathrm{I}}=2$, relay density is varied over the range $\rho_{\mathrm{R}} \in[1,100]$ and the infrastructure spatial weighting factor is varied over the range $\alpha \in\{0.1,1,10\}$. Results from Monte Carlo simulations are included to validate the analysis. The plot clearly shows that infrastructure spatial bias influences outage performance and that a homogeneous spatial bias is non-optimum. The plot also demonstrates the tightness of the upper bound.

Thus far, we have only considered the case for fixed terminals. We now proceed to average (8), and the upper bound (10), over the locations of uniformly distributed terminal nodes confined within $\mathcal{V}$. We denote these averages by $\overline{\mathcal{O}}$ and $\overline{\mathcal{O}}^{\mathrm{UB}}$ respectively, where

$$
\overline{\mathcal{O}}:=\mathbb{E}_{\mathbf{p}_{1}, \mathbf{p}_{2}}\left[\overline{\mathcal{O}}_{12}\right]=\frac{1}{V^{2}} \int_{\mathcal{V}^{2}} \overline{\mathcal{O}}_{12} \mathrm{~d} \mathbf{p}_{1} \mathrm{~d} \mathbf{p}_{2}
$$

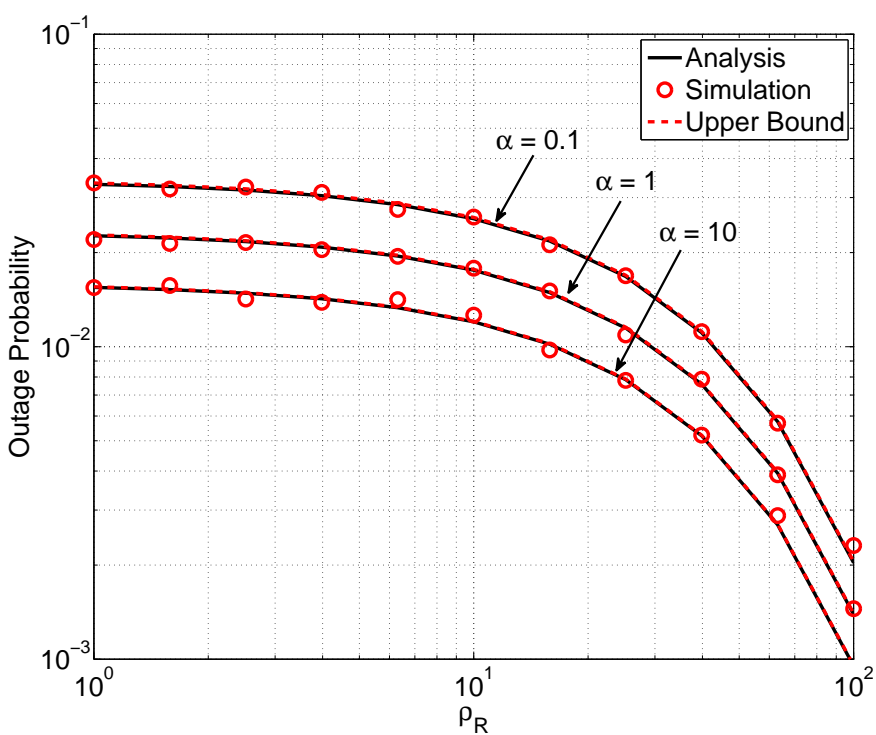

Fig. 5. Outage probability as a function of $\rho_{\mathrm{R}}$, averaged over locations of relay and infrastructure nodes over a circular domain $\mathcal{V}$ of radius 2.5, as given by (8), with appropriate substitutions. Terminal node locations are both fixed at a radial offset of 2 with angular separation of $\pi / 2$ and mean infrastructure density is $\rho_{\mathrm{I}}=2$. Plot illustrates outage probability over a range of infrastructure spatial weighting factors $\alpha \in\{0.1,1,10\}, \beta=2$ and includes results from Monte Carlo simulations. Dashed red lines indicate upper bounds derived from the application of the union bound of outage probabilities between terminal nodes and infrastructure nodes.

$$
\overline{\mathcal{O}}^{\mathrm{UB}}:=\mathbb{E}_{\mathbf{p}_{1}, \mathbf{p}_{2}}\left[\overline{\mathcal{O}}_{12}^{\mathrm{UB}}\right]=\frac{1}{V^{2}} \int_{\mathcal{V}^{2}} \overline{\mathcal{O}}_{12}^{\mathrm{UB}} \mathrm{d} \mathbf{p}_{1} \mathrm{~d} \mathbf{p}_{2}
$$

It should be noted that (25) and (26) apply to both homogeneous and inhomogeneous cases with appropriate substitutions for $\overline{\mathcal{O}}_{12}$ and $\overline{\mathcal{O}}_{12}^{\mathrm{UB}}$. Since these expression do not produce closed form solutions, we solve numerically by employing the polar coordinate system for $\mathbf{p}_{1}$ and $\mathbf{p}_{2}$ and using Riemann integration. We illustrate the relationship with $\alpha$ in Fig. 6, for a range of domain sizes. We observe a quasi-convex relationship where the value of $\alpha$ corresponding to the global minimum reduces as the domain size grows, approaching unity in the limit $R \rightarrow \infty$. Intuitively, as the domain size grows, the average separation of terminal nodes is such that direct connection, or connection via another relay node, occurs with low probability and connectivity is therefore dominated by the infrastructure. Since terminal nodes are most likely to connect to their proximity infrastructure nodes then a uniform spatial distribution is clearly optimal for larger domain sizes. This can be proven by considering the dominant terms of (8) when $r_{12} \gg 1$, and equating their 1 st derivatives to zero, which yields the condition that $\alpha=1$. A very tight upper bound is also observed which simplifies subsequent analysis of quasi-convexity in $\alpha$, as detailed in Appendix B. Although a robust mathematical proof of quasi-convexity is generally not possible in this case, the homogeneity of the averaging process (over the terminal and relay node positions) intuitively suggests that such a condition is true, and an extensive simulation study has supported this hypothesis. In subsequent analysis we assume quasi-convexity. 


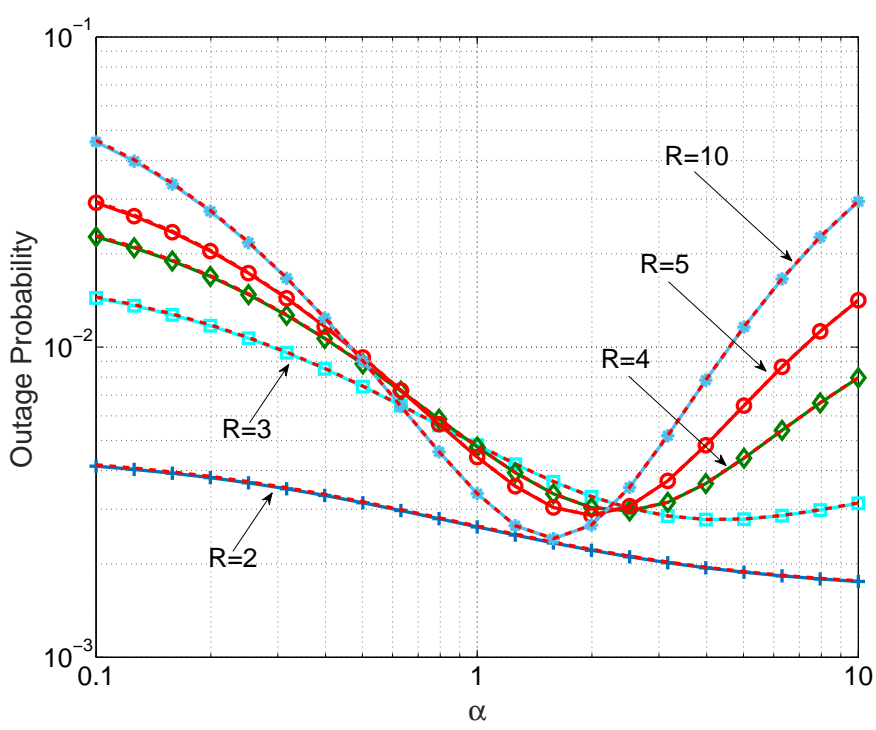

Fig. 6. Outage probability, averaged over relay, infrastructure and terminal node locations, as a function of weighting factor $\alpha$ for a range of domain radii $R \in\{2,3,4,5,10\}$. In all cases, mean densities of relay and infrastructure networks are fixed at $\rho_{\mathrm{R}}=50$ and $\rho_{\mathrm{I}}=2.5$. Dashed red lines indicate upper bounds derived from the application of the union bound of outage probabilities between terminal nodes and infrastructure nodes averaged over terminal node locations.

\section{NUMERICAL ANALYSIS OF RELATIVE DENSITIES OF RELAY AND INFRASTRUCTURE NETWORKS}

We now consider how our analysis may be applied in a 'real-world' context. By way of example, we may consider cellular $^{3}$ or vehicular networks, which typically have fixed infrastructures and where the locations and density of mobile phones, or vehicles, vary as they move, enter and leave the network. Since service providers generally have full or limited control over the network infrastructure, we may wish to determine the minimum infrastructure density and associated spatial distribution that achieves a minimum acceptable quality of service (QoS) from the composite network at a given average density of the relay (mobiles or vehicles) network. The infrastructure can then be controlled dynamically according to the temporal behaviour of the relay network.

In order to provide this behavioural insight, we equate (25) to a fixed prescribed outage probability $\kappa \in[0,1]$. In subsequent analysis we assume that $\kappa=0.01$ provides an acceptable QoS. Understanding the infrastructure/relay density relationship provides an insight into possible infrastructure activation/deactivation strategies, as relay nodes enter or leave the network. We evaluate (25) numerically, following substitutions of (8), (23) and (24), and employ Newton's method of successive approximation to yield $\rho_{\mathrm{I}}$, for a given $\rho_{\mathrm{R}}$. In reality, this approach would be significantly less computationally process intensive than employing a Monte Carlo process. In order to illustrate the effect of infrastructure spatial distribution bias as the domain size increases, we consider a range of biases $\alpha \in\{0.1,0.5,1.0,2.0,10\}$ and domain sizes

${ }^{3}$ Although interference would need to be considered in this case.
$R \in\{1,1.4,1.8,2.5,4,5\}$. The results of this analysis are illustrated in Fig. 7. Each trace represents a Pareto frontier in the average relay and infrastructure node densities, which vary with spatial distribution of the infrastructure network. With knowledge of the average relay density, we can actively reduce or increase the average infrastructure density, by selective deactivation or activation of infrastructure nodes, in order to maintain or exceed our prescribed outage condition; provided the densities lie on or above the Pareto frontier.

Remark (Deactivation Strategy). Selective deactivation of infrastructure nodes is equivalent to the aforementioned thinning of our point process $\Phi_{I}$, such that the resultant PPP, representing the infrastructure, has a spatial distribution with weighting factor $\alpha$. With a 'real-world' network with a finite number of infrastructure nodes at fixed locations, we may employ an infrastructure deactivation strategy that results in a spatial configuration of infrastructure nodes yielding the closest approximation to this optimal spatial distribution, defined by $\alpha$ and $\beta$, and the required average density $\rho_{I}$.

We observe, that for lower domain sizes, $R<2$, appreciable rates of reduction of infrastructure density, with increasing relay density, can be realised to the point whereby the relay density is sufficient to establish the required outage conditions without any infrastructure network. For the cases where $R<1.4$ and with no relay nodes, a lower density of infrastructure nodes are required when they are spatially biased towards the centre. As the relay density increases, this spatial bias becomes less important. This implies that, as the density of relay nodes increases, infrastructure nodes closer to the boundary should be deactivated in the thinning process. We observe a transition at a radius $R=1.4$, whereby for increasing radii, lower infrastructure densities are required when spatially biased towards the boundary.

Remark (Transition Radius). If we assume a fixed infrastructure network density, the expected number of infrastructure nodes will reduce with domain size. For smaller domain sizes (when the domain radius is less than or close to the hard connection range $r_{0}$ ) and with the absence of relay nodes, a randomly located terminal node is likely to have more infrastructure nodes within connection range when they are located towards the centre of the domain. As domain size grows above $r_{0}$ and with infrastructure nodes centrally biased there will be regions where terminal nodes are located closer to the boundary that will have limited or no access to any infrastructure node, thus requiring an increased spatial bias toward the domain boundary.

As the domain size increases beyond $R=1.8$, the rate of reduction of infrastructure density, with increasing relay density, is significantly lower and the spatial bias of the infrastructure has a more marked effect on performance, with an optimum spatial bias occurring at approximately $\alpha=2$ for $R=5$.

We now focus on the optimum spatial distribution for the infrastructure network, denoted by $\alpha_{\mathrm{opt}}$, that yields a minimum in averaged outage probability as defined by (25). Since we conjecture a quasi-convex relationship in (25) with respect to 

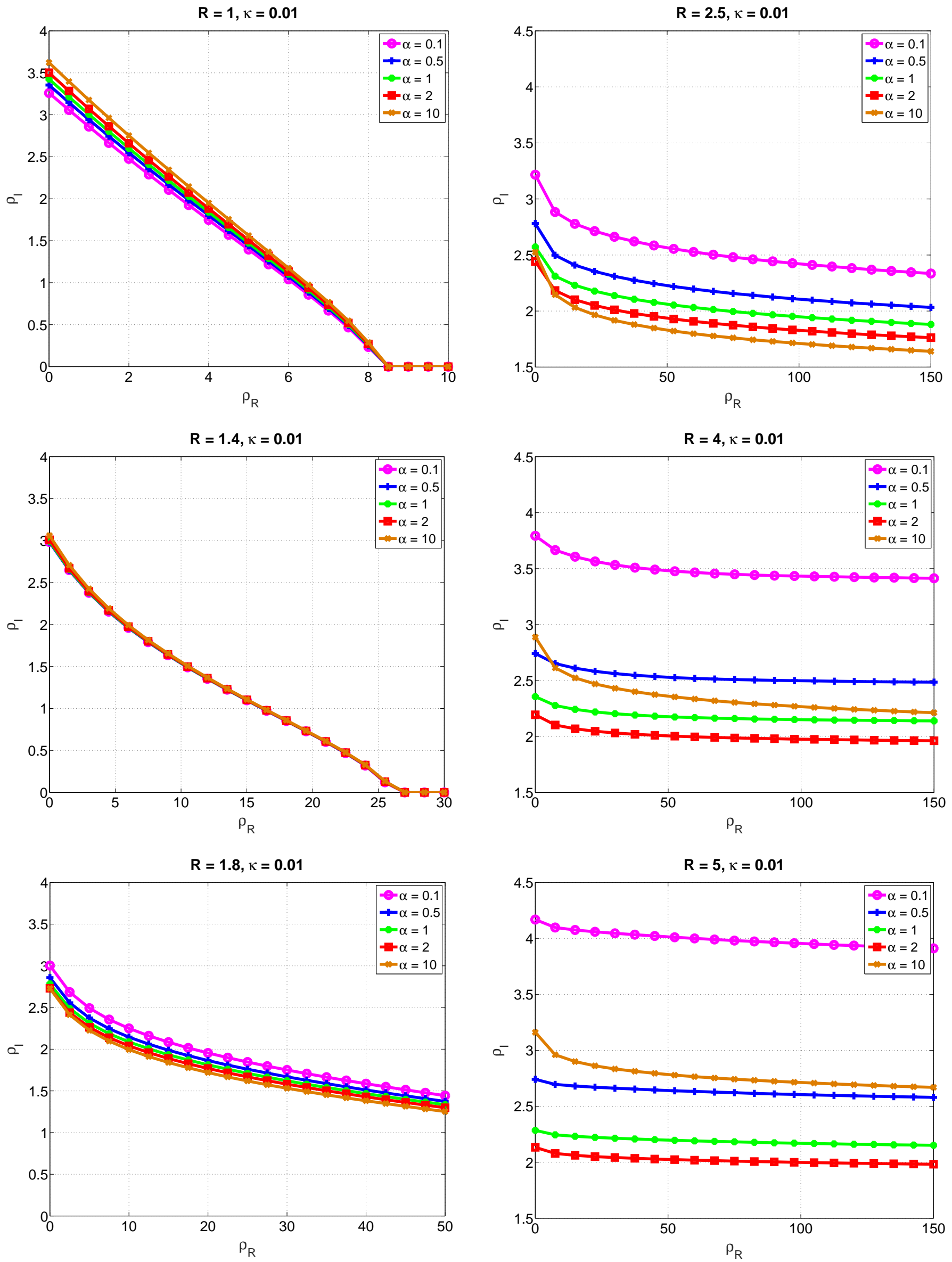

Fig. 7. Infrastructure density, $\rho_{\mathrm{I}}$, versus relay density, $\rho_{\mathrm{R}}$, achieving an outage probability of $\kappa=0.01$ averaged over positions of the terminal nodes over circular domain of radii $R \in\{1,1.4,1.8,2.5,4,5\}$ and infrastructure spatial distribution weighting $\alpha \in\{0.1,0.5,1,2,10\}$. 


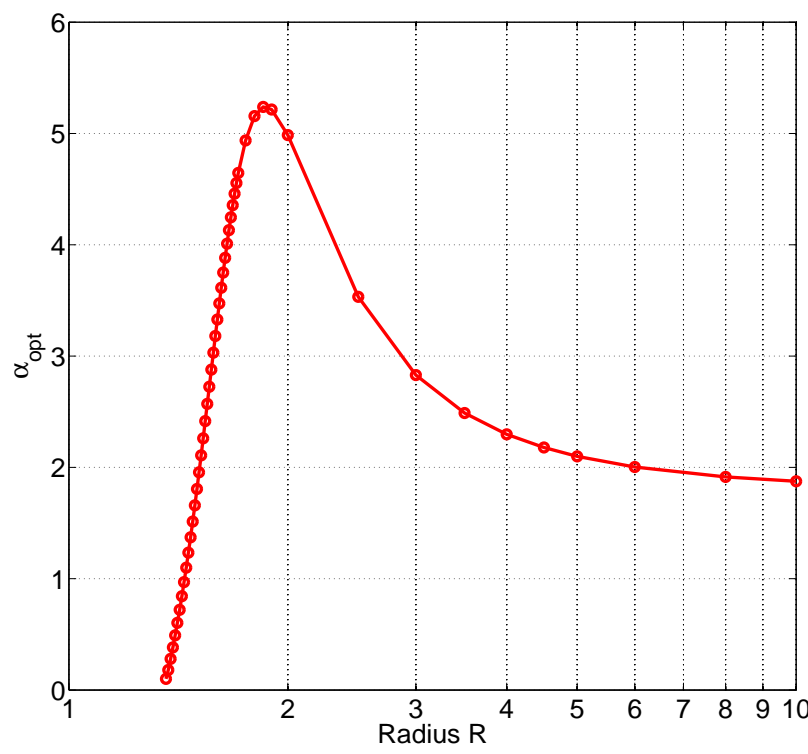

Fig. 8. For fixed infrastructure density of $\rho_{\mathrm{I}}=3$ and no relay network, we show the optimum spatial bias $\alpha_{\text {opt }}$ (minimising averaged outage probability) as a function of domain radius $R$.

$\alpha$, we solve

$$
\frac{\partial \overline{\mathcal{O}}}{\partial \alpha}=\frac{1}{V^{2}} \int_{\mathcal{V}^{2}} \frac{\partial \overline{\mathcal{O}}_{12}}{\partial \alpha} \mathrm{dp}_{1} \mathrm{~d} \mathbf{p}_{2}=0
$$

by employing Newton's method of successive approximation. The derivatives of $\overline{\mathcal{O}}_{12}$, with respect to $\alpha$ are relatively straightforward and not included for brevity. We firstly consider the optimum spatial distribution of a fixed density infrastructure, in the absence of relay nodes, as a function of domain size. This is illustrated in Fig. 8 for an infrastructure density $\rho_{\mathrm{I}}=3$ and zero relay density. We show that for increasing radius, we firstly observe the sharp transition from a centrally biased to boundary biased infrastructure; we remark on this transition radius earlier. The spatial bias then reaches a maximum at $R \approx 1.9$, followed by an asymptotic decay towards a uniform spatial distribution. This decay is an intuitive result since, as we approach a network of infinite extent, a uniformly distributed infrastructure is required to provide coverage over the whole domain.

We now proceed to investigate both the minimum infrastructure density, denoted by $\rho_{\mathrm{I}_{\min }}$, and the optimum spatial infrastructure bias, that achieves the prescribed averaged outage probability $\overline{\mathcal{O}}=\kappa$. We evaluate these over a range of relay densities and domain radii using the following process:

1) Fix domain radius $R$ and initially set $\rho_{\mathrm{R}}=0$ and $\alpha=$ $\alpha_{\mathrm{opt}}=1$.

2) Substitute $\left(R, \rho_{\mathrm{R}}, \alpha\right)$ into (25) and determine $\rho_{\mathrm{I}_{\mathrm{min}}}$, satisfying $\overline{\mathcal{O}}=\kappa$, by successive approximation.

3) Substitute $\left(R, \rho_{\mathrm{I}}=\rho_{\mathrm{I}_{\text {min }}}\right)$ into (27) and determine $\alpha_{\text {opt }}$ by successive approximation.

4) Due to interdependence of $\alpha$ and $\rho_{\mathrm{I}}$, iterate steps 2 and 3 until an acceptably low difference (e.g. 1\%) is reached between successive approximations of $\alpha$ and $\rho_{\mathrm{I}}$; we find 2 iterations are satisfactory.
5) Increment $\rho_{R}$ and repeat from step 2 using previous the previous $\rho_{\mathrm{I}_{\min }}$ and $\alpha_{\mathrm{opt}}$ to seed the subsequent successive approximations.

We focus on the larger domains with $R \in\{2.5,3,4\}$, since the reduction in infrastructure with spatial bias compared to the uniform distribution case, for lower domain sizes, is negligible. Results are shown in Fig. 9 which includes the density relationship for the uniform $\alpha=1$ case. Firstly, we observe that employing a non-uniform spatial distribution of infrastructure can realise significant reductions in the infrastructure density compared to the uniformly distributed case, as illustrated in Fig. 10, where reductions between $8 \%$ and $16 \%$ are observed. Secondly, if we consider the relationship between optimum $\alpha$, denoted by $\alpha_{\text {opt }}$, and relay density, we observe that monotonicity of the slopes of the traces change from strictly increasing to decreasing as the domain size increases.

Remark (Optimum Spatial Bias). The analysis for lower domain sizes, above the aforementioned transition radius but with $R<3$, shows that, with increasing relay density, infrastructure nodes closest to the domain centre should be deactivated and remaining active infrastructure nodes should be strongly concentrated at the boundary region. This suggests that the relay network dominates connectivity within the bulk and infrastructure nodes at the boundary compensate for loss of relay connectivity mass towards the boundary region. This dominance decreases with increasing domain size and we observe that $\alpha_{\text {opt }}$ reduces, an intuitive result since as we approach a network of infinite extent, a uniformly distributed infrastructure is required.

\section{CONClusion And Discussions}

In this paper, we have studied the connectivity between two terminal nodes employing direct, 2-hop and infrastructure based connectivity within a circular domain subject to Rayleigh fading. We derive mathematical expressions involving well-understood special functions for outage probability and show a dependence on: domain size, spatial separation of terminal nodes, their mid-point location and the densities of the relay and infrastructure networks. Our analysis has demonstrated that 2-hop and infrastructure connectivity performance reduces towards the boundaries, which agrees with previous observations [18]. With the objective of minimising outage probability between two randomly selected independent uniformly distributed (i.u.d.) terminal nodes, we show that employment of direct and relay assisted connectivity can significantly reduce the reliance on the infrastructure. Furthermore, the spatial distribution of infrastructure nodes has a marked bearing on connectivity performance in the finite domain regime, opening up the possibility of spatially selective deactivation of infrastructure nodes conditioned on relay density. We provide a mathematical framework that enables the determination of the optimal density and spatial distribution of an infrastructure network, for a given relay density and prescribed outage condition. As the domain sizes increase, we observe diminishing returns in employing direct and relay assisted connectivity due to increased average separation of 

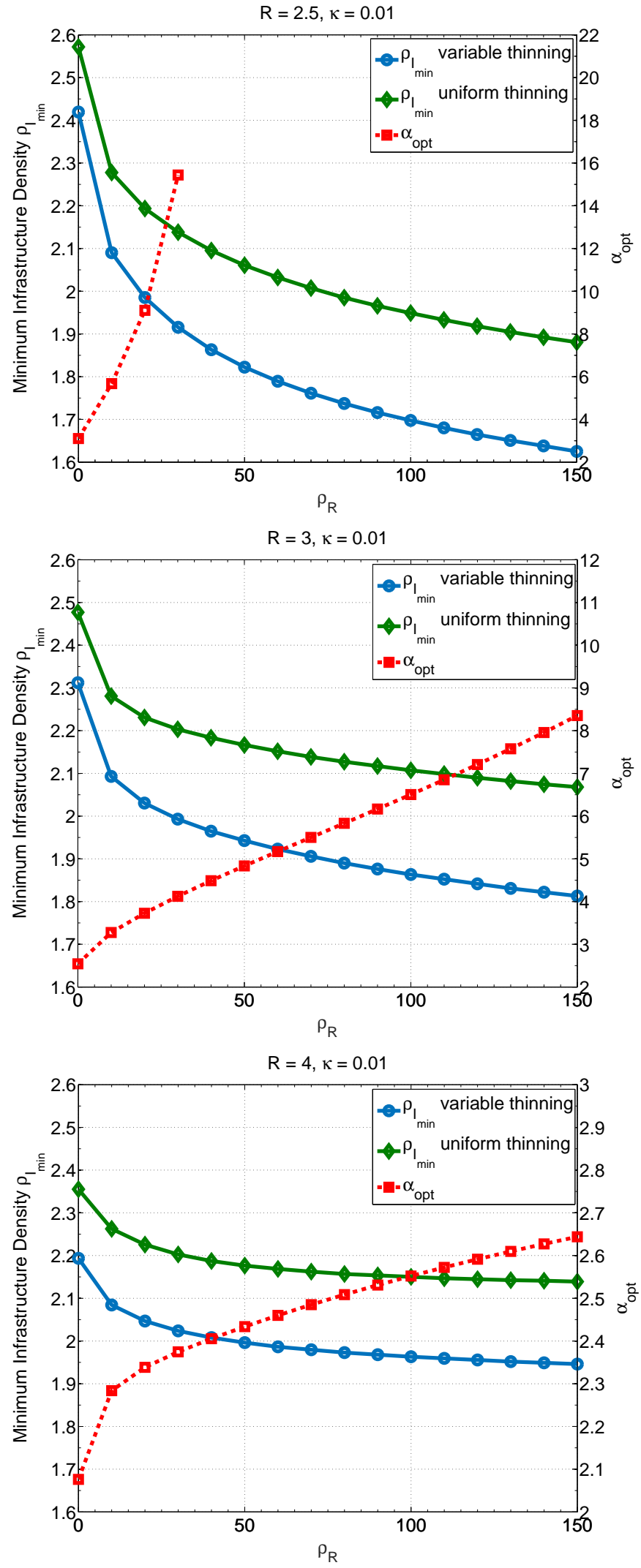

Fig. 9. Minimum infrastructure density, employing variable (non uniform) and uniform thinning, and optimum infrastructure spatial bias $\alpha_{\mathrm{opt}}$ as a function of relay density for radii $R \in\{2.5,3,4\}$.

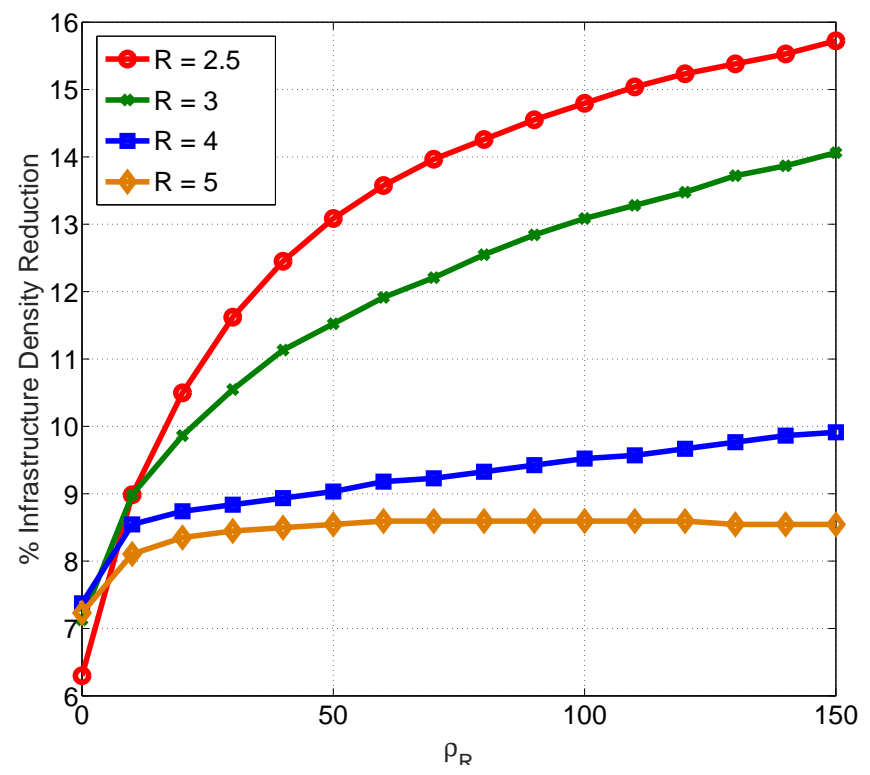

Fig. 10. Reduction in infrastructure density by employment variable (non uniform) spatial thinning relative to the uniform spatial thinning case.

terminal nodes and therefore an increased reliance on the infrastructure, which could be addressed by allowing 2-hop connectivity between terminal and infrastructure nodes via an intermediate relay node; a natural extension to this analysis.

$$
\begin{gathered}
\text { Appendix A } \\
\text { Evaluation of } \bar{H}_{12}^{\left(2_{\mathrm{I}}\right)} \\
\bar{H}_{12}^{\left(2_{\mathrm{I}}\right)}=\int_{\mathcal{V}} g\left(\mathbf{p}_{n}\right) H_{1 n} H_{2 n} \mathrm{~d} \mathbf{p}_{n} \\
=\int_{0}^{R} \int_{0}^{2 \pi} r_{n} g\left(\mathbf{p}_{n}\right) e^{-\left(\left\|r_{1 n}\right\|^{\eta}+\left\|r_{2 n}\right\|^{\eta}\right)} \mathrm{d} \phi_{n} \mathrm{~d} r_{n},
\end{gathered}
$$

where $g\left(\mathbf{p}_{n}\right)$ is given by (20). For mathematical tractability, we assume a path loss exponent $\eta=2$ and a quadratic spatial distribution function for $\mathbf{p}_{n}$ i.e. $\beta=2$. Employing polar coordinate geometry, relative to the centre of the circle where $\mathbf{p}_{i}=r_{i} \angle \phi_{i}$, then

$$
r_{i n}=\sqrt{r_{i}^{2}+r_{n}^{2}-2 r_{i} r_{n} \cos \left(\phi_{n}-\phi_{i}\right)} .
$$

Substituting (29) into (28) and simplifying gives

$$
\begin{aligned}
\bar{H}_{12}^{\left(2_{\mathrm{I}}\right)} & =e^{-\left(r_{1}^{2}+r_{2}^{2}\right)} \int_{0}^{R} r_{n} g\left(\mathbf{p}_{n}\right) e^{-2 r_{n}^{2}} \\
& \times\left[\int_{0}^{2 \pi} e^{-2 r_{n}\left[r_{1} \cos \left(\phi_{n}-\phi_{1}\right)+r_{2} \cos \left(\phi_{n}-\phi_{2}\right)\right]} \mathrm{d} \phi_{n}\right] \mathrm{d} r_{n} .
\end{aligned}
$$

We now use the trigonometric identity

$$
r_{1} \cos \left(\phi_{n}-\phi_{1}\right)+r_{2} \cos \left(\phi_{n}-\phi_{2}\right)=\Psi \cos \left(\phi_{n}-\varphi\right),
$$

where

$$
\varphi=\tan ^{-1}\left[\frac{r_{1} \sin \left(\phi_{1}\right)+r_{2} \sin \left(\phi_{2}\right)}{r_{1} \cos \left(\phi_{1}\right)+r_{2} \cos \left(\phi_{2}\right)}\right]
$$


and

$$
\begin{aligned}
\Psi & =\sqrt{\left[r_{1} \cos \left(\phi_{1}\right)+r_{2} \cos \left(\phi_{2}\right)\right]^{2}+\left[r_{1} \sin \left(\phi_{1}\right)+r_{2} \sin \left(\phi_{2}\right)\right]^{2}} \\
& =\sqrt{r_{1}^{2}+r_{2}^{2}+2 r_{1} r_{2} \cos \left(\phi_{2}-\phi_{1}\right)} \\
& =\left\|\mathbf{p}_{1}+\mathbf{p}_{2}\right\| \\
& =2\left\|\overline{\mathbf{p}}_{12}\right\|
\end{aligned}
$$

We use $\overline{\mathbf{p}}_{12}=\left(\mathbf{p}_{1}+\mathbf{p}_{2}\right) / 2$ to represent the mid-point between $\mathbf{p}_{1}$ and $\mathbf{p}_{2}$. The inner integral within (30), denoted by $F\left(r_{n}\right)$, can now be written as

$$
F\left(r_{n}\right)=\int_{0}^{2 \pi} e^{-r_{n}\left\|\overline{\mathbf{p}}_{12}\right\| \cos \left(\phi_{n}-\varphi\right)} \mathrm{d} \phi_{n} .
$$

Since we integrate over $2 \pi$, the angular offset $\varphi$ can be ignored and (34) becomes

$$
\begin{aligned}
F\left(r_{n}\right) & =2 \int_{0}^{\pi} e^{-4 r_{n}\left\|\overline{\mathbf{p}}_{12}\right\| \cos \left(\phi_{n}\right)} \mathrm{d} \phi_{n} \\
& =2 \pi \mathrm{I}_{0}\left(4 r_{n}\left\|\overline{\mathbf{p}}_{12}\right\|\right),
\end{aligned}
$$

where $\mathrm{I}_{0}(a)=\frac{1}{\pi} \int_{0}^{\pi} e^{a \cos \theta} \mathrm{d} \theta$ is the zeroth order modified Bessel function of the first kind. Substituting (35) into (30) now gives

$$
\bar{H}_{12}^{\left(2_{1}\right)}=2 \pi e^{-\left(r_{1}^{2}+r_{2}^{2}\right)} \int_{0}^{R} r_{n} g\left(\mathbf{p}_{n}\right) e^{-2 r_{n}^{2}} \mathrm{I}_{0}\left(4 r_{n}\left\|\overline{\mathbf{p}}_{12}\right\|\right) \mathrm{d} r_{n} .
$$

Expanding $g\left(\mathbf{p}_{n}\right)$ and letting $r_{n}=\left\|\mathbf{p}_{n}\right\|$ gives

$$
\begin{aligned}
\bar{H}_{12}^{\left(2_{\mathrm{I}}\right)} & =\frac{2 \pi}{k} e^{-\left(r_{1}^{2}+r_{2}^{2}\right)}\left[\int_{0}^{R} r_{n} e^{-2 r_{n}^{2}} \mathrm{I}_{0}\left(4 r_{n}\left\|\overline{\mathbf{p}}_{12}\right\|\right) \mathrm{d} r_{n}\right. \\
& \left.+\left(\frac{\alpha-1}{R^{2}}\right) \int_{0}^{R} r_{n}^{3} e^{-2 r_{n}^{2}} \mathrm{I}_{0}\left(4 r_{n}\left\|\overline{\mathbf{p}}_{12}\right\|\right) \mathrm{d} r_{n}\right] .
\end{aligned}
$$

Applying [31, eqs. (10) \& (20)] to solve the integrals results in

$$
\begin{aligned}
\bar{H}_{12}^{\left(2_{\mathrm{I}}\right)} & =\frac{2 \pi}{k} e^{-\left(r_{1}^{2}+r_{2}^{2}\right)}\left\{\frac{1}{4} e^{2\left\|\overline{\mathbf{p}}_{12}\right\|^{2}}\left[1-\mathrm{Q}\left(2\left\|\overline{\mathbf{p}}_{12}\right\|, 2 R\right)\right]\right. \\
& +\left(\frac{\alpha-1}{8 R^{2}}\right)\left(2\left\|\overline{\mathbf{p}}_{12}\right\|+1\right) e^{2\left\|\overline{\mathbf{p}}_{12}\right\|^{2}} \\
& \times\left[1-\mathrm{Q}\left(2\left\|\overline{\mathbf{p}}_{12}\right\|, 2 R\right)\right] \\
& -\left(\frac{\alpha-1}{4}\right) e^{-2 R^{2}} \\
& \left.\times\left[\frac{\left\|\overline{\mathbf{p}}_{12}\right\|}{R} \mathrm{I}_{1}\left(4\left\|\overline{\mathbf{p}}_{12}\right\| R\right)+\mathrm{I}_{0}\left(4\left\|\overline{\mathbf{p}}_{12}\right\| R\right)\right]\right\}
\end{aligned}
$$

where $\mathrm{Q}_{1}(a, b)=\int_{b}^{\infty} x e^{-\frac{\left(x^{2}+a^{2}\right)}{2}} \mathrm{I}_{o}(a x) \mathrm{d} x$ is the first order Marcum-Q-function and where $\mathrm{I}_{1}(a)=\frac{1}{\pi} \int_{0}^{\pi} e^{a \cos \theta} \cos \theta \mathrm{d} \theta$ is the first order modified Bessel function of the first kind.
Factoring out $\frac{1}{4} e^{2\left\|\overline{\mathbf{p}}_{12}\right\|^{2}}$, recognising that $e^{2\left\|\overline{\mathbf{p}}_{12}\right\|^{2}-\left(r_{1}^{2}+r_{2}^{2}\right)}=$ $e^{\frac{1}{2} r_{12}^{2}}$ and simplifying, yields the final expression

$$
\begin{aligned}
\bar{H}_{12}^{\left(2_{\mathrm{I}}\right)} & =\frac{\pi}{2 k} e^{-\frac{1}{2} r_{12}^{2}} \\
& \times\left\{\left(\frac{(\alpha-1)\left(2\left\|\overline{\mathbf{p}}_{12}\right\|^{2}+1\right)}{2 R^{2}}+1\right)\right. \\
& \times\left[1-\mathrm{Q}_{1}\left(2\left\|\overline{\mathbf{p}}_{12}\right\|, 2 R\right)\right] \\
& -(\alpha-1) e^{-2\left(\left\|\overline{\mathbf{p}}_{12}\right\|^{2}+R^{2}\right)} \\
& \left.\times\left[\frac{\left\|\overline{\mathbf{p}}_{12}\right\|}{R} \mathrm{I}_{1}\left(4\left\|\overline{\mathbf{p}}_{12}\right\| R\right)+\mathrm{I}_{0}\left(4\left\|\overline{\mathbf{p}}_{12}\right\| R\right)\right]\right\} .
\end{aligned}
$$

\section{APPENDIX B \\ QUASI-CONVEXITY IN $\mathcal{O}$}

We investigate the quasi-convexity in the upper bound, as defined by (26), which expanded gives

$$
\begin{aligned}
\overline{\mathcal{O}}^{\mathrm{UB}} & =\frac{1}{V^{2}} \int_{\mathcal{V}^{2}}\left(1-H_{12}\right) \exp \left[-\rho_{\mathrm{R}} V \bar{H}_{12}^{\left(2_{\mathrm{R}}\right)}\right] \\
& \times\left\{\exp \left[-\rho_{\mathrm{I}} V \bar{H}_{1 \mathrm{I}}\right]+\exp \left[-\rho_{\mathrm{I}} V \bar{H}_{2 \mathrm{I}}\right]\right\} \mathrm{d} \mathbf{p}_{1} \mathrm{dp}_{2} .
\end{aligned}
$$

Since the probabilities of the $\mathbf{p}_{1}$ and $\mathbf{p}_{2}$ connecting to an infrastructure node will be identical when averaged over all possible terminal node locations in $\mathcal{V},(40)$ can be reduced to

$$
\begin{aligned}
\overline{\mathcal{O}}^{\mathrm{UB}} & =\frac{1}{V^{2}} \int_{\mathcal{V}^{2}} 2 s\left(\mathbf{p}_{1}, \mathbf{p}_{2}\right) \exp \left[-\rho_{\mathrm{I}} V \bar{H}_{1 \mathrm{I}}\right] \mathrm{d} \mathbf{p}_{1} \mathrm{~d} \mathbf{p}_{2} \\
& =\frac{2}{V} \int_{\mathcal{V}} s\left(\mathbf{p}_{1}\right) \exp \left[-\rho_{\mathrm{I}} V \bar{H}_{1 \mathrm{I}}\right] \mathrm{d} \mathbf{p}_{1}
\end{aligned}
$$

where $s\left(\mathbf{p}_{1}, \mathbf{p}_{2}\right)=\left(1-H_{12}\right) \exp \left[-\rho_{\mathrm{R}} V \bar{H}_{12}^{\left(2_{\mathrm{R}}\right)}\right]$ and $s\left(\mathbf{p}_{1}\right)=$ $\frac{1}{V} \int_{V} s\left(\mathbf{p}_{1}, \mathbf{p}_{2}\right) \mathbf{d} \mathbf{p}_{2}$, which is a non negative scaling factor independent of the infrastructure. We reduce (23) to the form

$$
\bar{H}_{1 \mathrm{I}}=\frac{A_{1} \alpha+B_{1}}{\alpha+1}, \quad \alpha>0
$$

where

$$
\begin{aligned}
A_{1} & =\frac{2}{R^{2}}\left\{\left(\frac{r_{1}^{2}+1}{R^{2}}\right)\left[1-\mathrm{Q}_{1}\left(\sqrt{2} r_{1}, \sqrt{2} R\right)\right]\right. \\
& \left.-e^{-\left(r_{1}^{2}+R^{2}\right)}\left[\frac{r_{1}}{R} \mathrm{I}_{1}\left(2 r_{1} R\right)+\mathrm{I}_{0}\left(2 r_{1} R\right)\right]\right\}, \\
B_{1} & =\frac{2}{R^{2}}\left\{\left(1-\frac{r_{1}^{2}+1}{R^{2}}\right)\left[1-\mathrm{Q}_{1}\left(\sqrt{2} r_{1}, \sqrt{2} R\right)\right]\right. \\
& \left.+e^{-\left(r_{1}^{2}+R^{2}\right)}\left[\frac{r_{1}}{R} \mathrm{I}_{1}\left(2 r_{1} R\right)+\mathrm{I}_{0}\left(2 r_{1} R\right)\right]\right\}
\end{aligned}
$$

and $r_{1}=\left\|\mathbf{p}_{1}\right\|$. We now define the exponential term within (41) as

$$
f_{a b}\left(\alpha, \mathbf{p}_{1}\right)=\exp \left[-\frac{a \alpha+b}{\alpha+1}\right],
$$

where $a=\rho_{\mathrm{I}} V A_{1}$ and $b=\rho_{\mathrm{I}} V B_{1}$. We now show that, by inspection of sub-level sets and super-level sets, that 
$f_{a b}\left(\alpha, \mathbf{p}_{1}\right)$ is quasi-linear in $\operatorname{dom} f_{a b}=\{\alpha \mid \alpha>0\}$. We let $S_{\varepsilon}$ denote a sub-level set, such that

$$
\begin{aligned}
S_{\varepsilon} & =\left\{\alpha \mid \alpha>0 ; \quad f_{a b} \leqslant \varepsilon\right\} \\
& =\{\alpha \mid \alpha>0 ; \quad a \alpha+b \geqslant-(\alpha+1) \ln \varepsilon\} \\
& =\left\{\alpha \mid \alpha>0 ; \quad\left\{\begin{array}{l}
\alpha \geqslant-\frac{b+\ln \varepsilon}{a+\ln \varepsilon}, a+\ln \varepsilon>0 \\
\alpha \leqslant-\frac{b+\ln \varepsilon}{a+\ln \varepsilon}, a+\ln \varepsilon<0
\end{array}\right\}\right.
\end{aligned}
$$

Since the intersection of an open convex set and a closed convex set is convex, quasi-convexity follows. The proof of quasi-concavity follows in an analogous manner using superlevel sets. Hence, we deduce the function is quasi-linear as stated. We can make further progress by considering monotonicity of the function $f_{a b}$. Inspection of (46) shows that $f_{a b}^{-1}$ is well defined on $(0, \infty)$. Hence, $f_{a b}$ is injective with strict monotonicity for all $a, b$ subject to $a \neq b$. Hence, $\overline{\mathcal{O}}^{\mathrm{UB}}$ is the limit of a positive weighted sum of strictly monotonic functions where the quasi-linear function $f_{a b}\left(\alpha, \mathbf{p}_{1}\right)$ is decreasing monotonic for $b / a<1$ and increasing monotonic for $b / a>1$. Denoting the 1st and 2nd derivatives of function $f_{a b}$ as $f_{a b}^{\prime}$ and $f_{a b}^{\prime \prime}$ respectively, where

$$
\begin{aligned}
& f_{a b}^{\prime}=-\frac{a-b}{(\alpha+1)^{2}} f_{a b} \\
& f_{a b}^{\prime \prime}=\left[2 \frac{a-b}{(\alpha+1)^{3}}+\frac{(a-b)^{2}}{(\alpha+1)^{4}}\right] f_{a b},
\end{aligned}
$$

we can show that when $b / a<1$, the decreasing monotonic is convex whereas, when $b / a>1$ the increasing monotonic is concave, under the condition $-2<(a-b)<0$ and quasi-linear (convex to concave with increasing $\alpha$ ) otherwise, with inflection point at $\alpha=-0.5(a-b)-1$. We illustrate the function $A_{1}-B_{1}$ with respect to $r_{1} / R$, for a range of domain radii, in Fig. 11, clearly showing a single zero crossing point for each radius. Denoting the radial offset at which the zero crossing occurs by $r_{t}$, we observe that $r_{t}$ can be approximated by the radius where the area of a circle of radius $r_{t}$ is approaching $50 \%$ of the total domain area $V$; it is relatively straightforward to show that with increasing $R$, $r_{t} \rightarrow 1 / \sqrt{2}$ and $A_{1}-B_{1} \rightarrow 0, \forall r_{1}$. In summary, $\overline{\mathcal{O}}^{\mathrm{UB}}$ will be sum of a strictly decreasing convex monotone and a strictly increasing quasi-linear function, in $\alpha$, which does not prove a uni-modal composite function. Nevertheless, the analysis presented here coupled with extensive numerical simulation suggests that quasi-convexity holds.

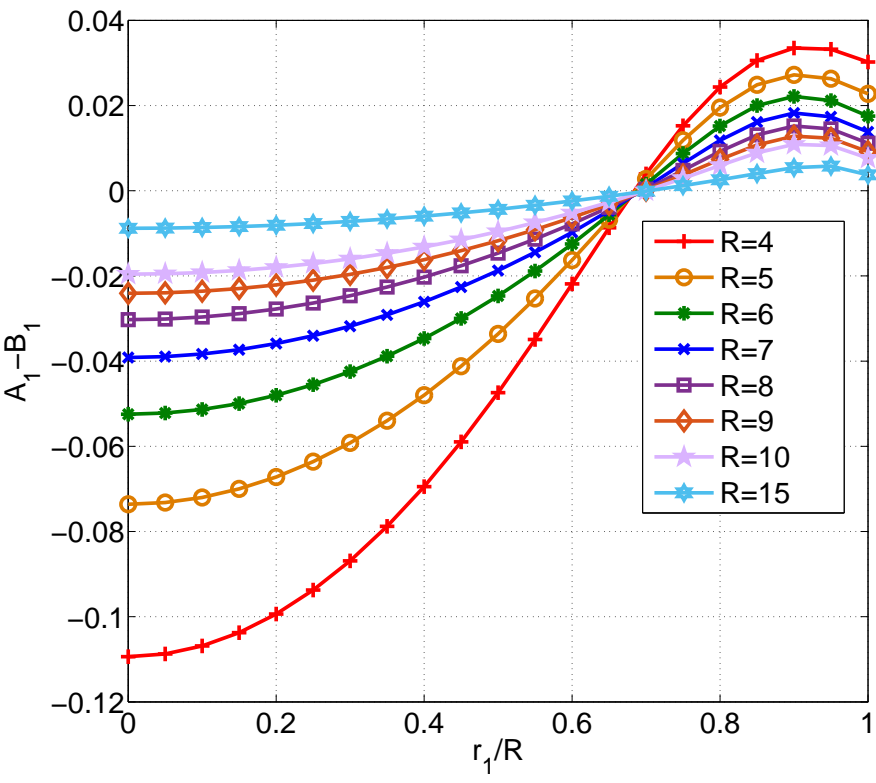

Fig. 11. Illustration of function $A_{1}-B_{1}$ with respect to $r_{1} / R$ for a range of domain radii $R \in\{6,7,8,9,10\}$. Showing a single zero crossing.

\section{REFERENCES}

[1] N. Bhushan, J. Li, D. Malladi, R. Gilmore, D. Brenner, A. Damnjanovic, R. Sukhavasi, C. Patel, and S. Geirhofer, "Network densification: the dominant theme for wireless evolution into 5G," IEEE Communications Magazine, vol. 52, no. 2, pp. 82-89, 2014.

[2] CISCO, "Cisco visual networking index: Global mobile data traffic forecast update, 2016-2021 white paper," Technical Report, Cisco, Tech. Rep., 2017.

[3] D. Lopez-Perez, M. Ding, H. Claussen, and A. H. Jafari, "Towards $1 \mathrm{Gbps} / \mathrm{UE}$ in cellular systems: Understanding ultra-dense small cell deployments," IEEE Communications Surveys Tutorials, vol. 17, no. 4, pp. 2078-2101, Fourthquarter 2015.

[4] X. An and F. Pianese, "Understanding co-channel interference in ltebased multi-tier cellular networks," in Proceedings of the 9th ACM symposium on Performance evaluation of wireless ad hoc, sensor, and ubiquitous networks. ACM, 2012, pp. 107-112.

[5] I. Hwang, B. Song, and S. S. Soliman, "A holistic view on hyperdense heterogeneous and small cell networks," IEEE Communications Magazine, vol. 51, no. 6, pp. 20-27, June 2013.

[6] J. G. Andrews, S. Buzzi, W. Choi, S. V. Hanly, A. Lozano, A. C. K. Soong, and J. C. Zhang, "What will 5G be?" IEEE Journal on Selected Areas in Communications, vol. 32, no. 6, pp. 1065-1082, June 2014.

[7] M. Ahsen, S. A. Hassan, and D. N. K. Jayakody, "Propagation modeling in large-scale cooperative multi-hop ad hoc networks," IEEE Access, vol. 4, pp. 8925-8937, 2016.

[8] J. Markendahl and . Mkitalo, "A comparative study of deployment options, capacity and cost structure for macrocellular and femtocell networks," in 2010 IEEE 21st International Symposium on Personal, Indoor and Mobile Radio Communications Workshops, Sept 2010, pp. $145-150$.

[9] K. Davaslioglu and E. Ayanoglu, "Quantifying potential energy efficiency gain in green cellular wireless networks," IEEE Communications Surveys Tutorials, vol. 16, no. 4, pp. 2065-2091, Fourthquarter 2014.

[10] Z. Hasan, H. Boostanimehr, and V. K. Bhargava, "Green cellular networks: A survey, some research issues and challenges," IEEE Communications Surveys Tutorials, vol. 13, no. 4, pp. 524-540, Fourth 2011.

[11] N. Yu, Y. Miao, L. Mu, H. Du, H. Huang, and X. Jia, "Minimizing energy cost by dynamic switching on/off base stations in cellular networks," IEEE Transactions on Wireless Communications, vol. 15, no. 11, pp. 7457-7469, 2016.

[12] A. Bousia, A. Antonopoulos, L. Alonso, and C. Verikoukis, "Green; distance-aware base station sleeping algorithm in LTE-Advanced," in 
2012 IEEE International Conference on Communications (ICC), June 2012, pp. 1347-1351.

[13] M. Ding, D. Lopez-Perez, G. Mao, and Z. Lin, "Performance impact of idle mode capability on dense small cell networks with los and nlos transmissions," arXiv preprint arXiv:1609.07710, 2016.

[14] M. Ding, D. L. Pérez, G. Mao, and Z. Lin, "Study on the idle mode capability with LoS and NLoS transmissions," arXiv preprint arXiv:1608.06694, 2016.

[15] A. Asadi, Q. Wang, and V. Mancuso, "A survey on device-to-device communication in cellular networks," IEEE Communications Surveys Tutorials, vol. 16, no. 4, pp. 1801-1819, Fourthquarter 2014.

[16] L. Wei, R. Hu, Y. Qian, and G. Wu, "Enable device-to-device communications underlaying cellular networks: challenges and research aspects," IEEE Communications Magazine, vol. 52, no. 6, pp. 90-96, June 2014.

[17] Networld, "White paper for research beyond 5G," Proceedings of the First International Conference on Communications and Networking in China (CHINACOM 2006).

[18] J. Coon, C. Dettmann, and O. Georgiou, "Full connectivity: Corners, edges and faces," Journal of Statistical Physics, vol. 147, no. 4, pp. 758-778, 2012. [Online]. Available: http://dx.doi.org/10.1007/s10955012-0493-y

[19] J. P. Coon, C. P. Dettmann, and O. Georgiou, "Connectivity of confined dense networks: boundary effects and scaling laws," arXiv preprint arXiv:1201.4013, 2012.

[20] D. A. Hedges, J. P. Coon, and O. Georgiou, "On the connectivity of two-hop networks in finite domains," in SCC 2017; 11th International ITG Conference on Systems, Communications and Coding, Feb 2017, pp. 1-6.

[21] M. Haenggi, J. G. Andrews, F. Baccelli, O. Dousse, and M. Franceschetti, "Stochastic geometry and random graphs for the analysis and design of wireless networks," IEEE Journal on Selected Areas in Communications, vol. 27, no. 7, pp. 1029-1046, September 2009.

[22] S. Kumar, V. S. Raghavan, and J. Deng, "Medium access control protocols for ad hoc wireless networks: A survey," Ad Hoc Networks, vol. 4, no. 3, pp. 326 - 358, 2006. [Online]. Available: http://www.sciencedirect.com/science/article/pii/S1570870504000873

[23] M. Haenggi, Stochastic Geometry for Wireless Networks, 1st ed. New York, NY, USA: Cambridge University Press, 2012.

[24] E. N. Gilbert, "Random plane networks," Journal of the Society for Industrial and Applied Mathematics, vol. 9, no. 4, pp. 533-543, 1961. [Online]. Available: http://dx.doi.org/10.1137/0109045

[25] O. Georgiou and C. Nguyen, "Multihop connectivity of ad hoc networks with randomly oriented directional antennas," Wireless Communications Letters, IEEE, vol. 4, no. 4, pp. 369-372, Aug 2015.

[26] O. Georgiou, C. P. Dettmann, and J. P. Coon, "Network connectivity: Stochastic vs. deterministic wireless channels," in 2014 IEEE International Conference on Communications (ICC), June 2014, pp. 77-82.

[27] J. Hu and N. C. Beaulieu, "Performance analysis of decode-and-forward relaying with selection combining," IEEE Communications Letters, vol. 11 , no. 6 , pp. 489-491, June 2007.

[28] J. P. Coon, O. Georgiou, and C. P. Dettmann, "Connectivity scaling laws in wireless networks," IEEE Wireless Communications Letters, vol. 4, no. 6, pp. 629-632, 2015.

[29] G. Amparo, S. Javier, and T. N. M., "The asymptotic and numerical inversion of the marcum qfunction," Studies in Applied Mathematics, vol. 133, no. 2, pp. 257-278. [Online]. Available: https://onlinelibrary.wiley.com/doi/abs/10.1111/sapm.12050

[30] D. Moltchanov, "Survey paper: Distance distributions in random networks," Ad Hoc Networks, vol. 10, no. 6, pp. 1146-1166, 2012.

[31] A. H. Nuttall, "Some integrals involving the Q-function," DTIC Document, Tech. Rep., 1972 